\title{
PERIODIC TRAJECTORIES OF A ONE-PARAMETER SEMIGROUP
}

\author{
BY F. BROCK FULLER
}

\author{
Communicated by Felix Browder, January 7, 1963
}

The object of Theorem 1 below is to establish the existence of periodic solutions of an autonomous differential equation $\dot{y}=f(y)$ by an extension of the Poincaré method of sections (see $[2 ; 4]$ ). The following situation is envisaged: the equation is defined on a subset $D$ of euclidean space and has unique solutions $y(x, t)$ jointly continuous in $t$ and the initial point $x ; D$ contains a compact subset $K$ with the property that the positive trajectories starting from points of $K$ remain in $K$. The assignment to $x$ in $K$ and $t$ in $[0, \infty)$ of the point $T_{t}(x)=y(x, t)$ in $K$ defines a continuous one-parameter semigroup $T_{t}$ acting on $K$, i.e., $T_{t}$ is jointly continuous in $x$ and $t, T_{0}$ is the identity on $K$ and $T_{s+t}=T_{s} \circ T_{t}$.

THEOREM 1. Let $K$ be a connected finite complex, let $T_{t}$ be a continuous one-parameter semigroup acting on $K$ and let $\omega$ be a closed 1-form on $K$ (defined over a portion of euclidean space containing $K$ ) with integervalued periods. Make the following two assumptions on $K, T_{t}$ and $\omega$ :

A. For each $x$ in $K$ there is a $t$ for which the integral of $\omega$ over the trajectory from $x$ to $T_{t}(x)$ is positive.

B. The classes of closed paths in $K$ over which the integral of $\omega$ vanishes form a subgroup of the fundamental group of $K$. Assume that the corresponding covering space $K^{i}$ has nonvanishing Euler characteristic.

Conclusion: $T_{t}$ has a periodic trajectory, i.e., there is an $x$ in $K$ and a period $p>0$ such that $T_{t+p}(x)=T_{t}(x)$ for all $t \geqq 0$.

REMARK a. If we denote the integral of $\omega$ over the trajectory from $x$ to $T_{t}(x)$ by $\Delta(x, t)$, assumption $A$ implies that there exists a positive constant $a$ such that $a t<\Delta(x, t)$ for sufficiently large $t$. Thus $\Delta(x, t)$ converges uniformly to $+\infty$. If $T_{t}$ is engendered by the differential equation $\dot{y}=f(y), \Delta(x, t)$ can be written as the integral with respect to $t$ of the scalar product $\omega \cdot f$, evaluated along the trajectory from $x$ to $T_{t}(x)$.

REMARK b. Although the covering space $K^{\prime}$ is not a finite complex, assumption $\mathrm{A}$ implies that $K^{\prime}$ has finite Betti numbers, so that its Euler characteristic is defined.

REMARK c. The period of the periodic trajectory disclosed by the theorem is bounded by a number depending on the uniform rate of 
convergence of $\Delta(x, t)$ to $+\infty$, the periods of $\omega$ and the Betti numbers of $K^{\prime}$.

By means of the construction outlined in [2], Theorem 1 can be derived from the following theorem.

TheOREM 2. Let $F$ be an upper semicontinuous multiple-valued function from a finite complex $X$ into itself. Let the system of endomorphisms $F_{* p}$ of $H_{p}(X)$, the homology groups of $X$ with real coefficients, be induced by $F$. Denote by $r_{p}$ the lowest value to which rank $F_{* p}^{k}$ descends as $k$ increases. Then $\sum(-1)^{p} r_{p} \neq 0$ implies that $F$ has a periodic point: $x \in F^{N}(x)$ and the period $N$ does not exceed the larger of $\sum r_{2 q}$ and $\sum r_{2 q+1}$.

The proof of Theorem 2, using the Lefschetz formula for multiplevalued functions $[4 ; 5]$ is essentially the same as that of the more special theorem in [1].

The relationships in Theorem 1 are illustrated by the following construction. Let $f$ be any continuous mapping of a connected finite complex $X$ into itself. The mapping cylinder $C_{f}$ of $f$, constructed using two copies of $X$, one for the domain and one for the range of $f$, can be made into a space $K$ by identifying the two copies. A semigroup $T_{t}$ acting on $K$ is obtained by moving all points at a uniform rate along the segments from $x$ to $f(x)$. A closed 1 -form $\omega$ with integer periods can be defined on $K$ which is zero on the subspace $X$ and such that the integral of $\omega$ over any segment from $x$ to $f(x)$ is +1 ; $\omega$ satisfies assumption $\mathrm{A}$. The covering space $K^{\prime}$ is then a space obtained by coupling together copies $C_{f}^{n},-\infty<n<+\infty$, of $C_{f}$. For the endomorphism $f_{* p}$ of $H_{p}(X)$, the integer $r_{p}$ defined in the statement of Theorem 2 turns out to be the pth Betti number of $K^{\prime}$, so that by Theorem 2 nonvanishing of the Euler characteristic of $K^{\prime}$ (assumption B) implies that $f$ has a periodic point and $T_{t}$ a periodic trajectory.

A proof of Theorem 1 will appear elsewhere.

\section{REFERENCES}

1. F. B. Fuller, The existence of periodic points, Ann. of Math. (2) 57 (1953), 229-230.

2. - Fixed points of multiple-valued transformations, Bull. Amer. Math. Soc. 67 (1961), 165-169. 1957.

3. S. Lefschetz, Differential equations: geometric theory, Interscience, New York,

4. - On coincidences of transformations, Bol. Soc. Mat. Mexicana 2 (1957), 16-25.

5. B. O'Neill, Induced homology homomorphisms for set-valued maps, Pacific J. Math. 7 (1957), 1179-1184.

California Institute of Technology 